\title{
Aproximación al establecimiento de una red de referencia para la implantación de la Directiva Marco del Agua en la Comunidad de Madrid
}

\author{
Alicia Bordallo López ${ }^{1, *}$ y Carmen Casado Sancho ${ }^{2, *}$ \\ Departamento de Ecología, Facultad de Ciencias. Universidad Autónoma de Madrid. Cantoblanco, 28049 \\ Madrid. Spain.
}

* Autor responsable de la correspondencia: aliciabor@gmail.com

Recibido: 28/9/2010

Aceptado: 9/6/2011

\begin{abstract}
Approximation to the establishment of a reference network for the establishment of the Water Framework Directive in the Community of Madrid

This article presents the results of the search for stations that can constitute a reference network to evaluate the ecological quality of the rivers in the Community of Madrid (Central Spain). Eleven river typologies were defined based primarily on their lithology and morphology. By means of the map of uses and eliminating zones affected by anthropic usage, 16 preliminary reference stations were selected, that were visited to check if they showed local disturbances that could prevent them from being considered reference stations. Seven sites were kept: three of which are of a siliceous mountain river typology and four of which are of a piedmont siliceous river typology. For the rest of the typologies, ecological potentials must be defined or reference points outside the study area must be found. In these stations diverse physical-chemical parameters were measured and the IBMWP, IASPT, QBR, RQI, IHF indices were calculated.

Only the station located in the Umbría Stream presented the adequate values of all the indices to be considered a reference station. The stations located in the Guadarrama, Navacerrada, Manzanares, Madarquillos Rivers and in the Mediano Stream presented a high concentration of phosphates, due to their natural characteristics, which allows them also to be considered as reference stations, which excludes only the station located in the Perales River. We concluded that it is difficult to create a reference network that allows an ecological evaluation of the rivers in the Community of Madrid because there are few points that meet the characteristics indicated to be considered natural or nearly natural rivers.
\end{abstract}

Key words: Reference, typologies, biotic indices, physical-chemical variables.

\section{RESUMEN}

Aproximación al establecimiento de una red de referencia para la implantación de la Directiva Marco del Agua en la Comunidad de Madrid

En este trabajo se presentan los resultados de la búsqueda de estaciones que pueden constituir una red de referencia para evaluar la calidad ecológica de los ríos de la Comunidad de Madrid (España Central). Se definieron once tipologías de río basándose principalmente en litología y morfología. Empleando el mapa de usos y descartando zonas afectadas por usos antrópicos, se seleccionaron 16 estaciones preliminares de referencia, que fueron visitadas para comprobar si presentaban afecciones locales que impidieran considerarlas estaciones de referencia. Se mantuvieron siete puntos: tres de la tipología de ríos montañosos silíceos y cuatro de la tipología de pie de monte silíceo; para el resto de tipologías se deberán definir potenciales ecologicos o buscar puntos de referencia fuera del área de estudio. En estas estaciones se midieron diversos parámetros físico-químicos y se calcularon los índices IBMWP, IASPT, QBR, RQI, IHF.

Sólo la estación ubicada en el Arroyo Umbría presentó valores adecuados de todos los índices para ser considerada de referencia. Las estaciones ubicadas en los ríos Guadarrama, Navacerrada, Manzanares, Madarquillos y en el Arroyo Mediano presentaron una concentración elevada de fosfatos, debida a sus características naturales, lo que permite considerarlas también como estaciones de referencia y excluir solamente la estación ubicada en el río Perales. Concluimos que es difícil crear una red de referencia que permita evaluar ecológicamente los ríos de la Comunidad de Madrid porque existen pocos puntos que cumplan las características indicadas para ser considerados naturales o cercanos a la naturalidad.

Palabras clave: Referencia, tipologías, índices bióticos, variables físico-químicas. 
Bordallo y Casado

\section{INTRODUCCIÓN}

El objetivo perseguido por la Directiva Marco del Agua (Directiva 2000/60/CE; DMA) es prevenir el deterioro de los ecosistemas acuáticos, obligando a los estados miembros a conseguir que dichos ecosistemas alcancen al menos un buen estado ecológico en el año 2015. Para controlar el paulatino cumplimiento de los requisitos de la DMA, se demanda la creación de una serie de redes de control y gestión del estado ecológico. Sin embargo, para lograr este objetivo en primer lugar hay que establecer unas clases de Estado Ecológico en función de criterios biológicos, hidrológicos y físico-químicos, clases que deben definirse en base a unas estaciones de referencia localizadas en tramos de río con escasa o nula alteración, situadas en los diferentes ecotipos fluviales que previamente se tienen que haber definido de acuerdo a las directrices de la propia DMA.

En España se han realizado diferentes estudios para definir la tipología de los ríos atendiendo a la DMA, en primer lugar el realizado por Bonada et al. (2002a) a partir del sistema B de la DMA. Posteriormente el Centro de Estudios y Experimentación de Obras Públicas (CEDEX) realizó una tipología de ríos para la península ibérica (CEDEX, 2005), basándose en una regionalización previa de la cuenca del Ebro realizada adoptando los sistemas estadísticos de clasificación de las variables ambientales. Para elaborar la tipología del CEDEX se utilizaron variables que englobaban aspectos relacionados con características hidrológicas, bioclimáticas, geológicas y morfométricas de las cuencas vertientes, definiéndose finalmente 32 tipos de tramos fluviales. González del Tánago y García de Jalón (2006) realizan otra propuesta partiendo desde la ecorregión en la que se encuentra la cuenca hidrográfica y la provincia biogeográfica a la que pertenece (Rivas Martinez et al., 2002), y considerando las zonas de vegetación de ribera de la península ibérica (Lara, 2005) así como el tamaño y la geología de la cuenca vertiente, hasta variables a escala de segmento fluvial como el régimen de caudales, el tipo geomorfológico de cauce (adaptado de Rosgen, 1996), el tipo de valle y variables del hábitat fluvial.
A otra escala espacial, en la Comunidad de Madrid existe una red para el control de la calidad del agua (red ICA), además de las estaciones de control biológico de la Confederación Hidrográfica del Tajo. Pero dichas redes de control no son válidas para establecer la red de estaciones de referencia de la Comunidad porque incumplen los criterios que la DMA impone: conservación del bosque de ribera, naturalidad del cauce fluvial, ausencia de embalses, hábitat del lecho adecuado y concentraciones bajas de los parámetros físico-químicos. El objetivo de este trabajo es establecer una red de estaciones de referencia para poder evaluar la calidad ecológica de los ríos de la Comunidad. Para ello esta red debe disponer de estaciones en los ríos que discurren por la Comunidad que se consideren de buena calidad ecológica para un determinado tipo fluvial, con unas características concretas que se empleen para definir tipologías.

\section{MATERIAL Y MÉTODOS}

\section{Tipología de los ríos de la Comunidad de Madrid y selección preliminar de estaciones}

La Comunidad Autónoma de Madrid tiene una superficie aproximada de $8000 \mathrm{~km}^{2}$ y se haya en la vertiente suroeste de la Cordillera Central. Sus aguas pertenecen a la Cuenca del Tajo. La cota más elevada se encuentra en Peñalara (2.429 m) y la más baja en el Alamín (430 m). El desnivel que hay entre estos puntos hace que la $\mathrm{Co}-$ munidad cuente con una gran riqueza ecológica (Carranza \& Arístegui, 2002).

Desde el punto de vista geomorfológico está dividida en dos dominios geológico-estructurales: la Sierra de Guadarrama y la Depresión del Tajo. La sierra pertenece al Sistema Central, su litología es granítica. La vegetación arbórea está constituida principalmente por pinos, robles, encinas y enebros (Carranza \& Arístegui, 2002). En la depresión del Tajo hay cuatro tipos de estructuras geológicas (Carranza \& Arístegui, 2002): 1) las arenas, conglomerados y arcillas con cultivos de cereal de secano y pastizal; 2) los yesos, arcillas y margas yesíferas de la zona cen- 
tral de la Depresión con cultivos de cereal, viña $\mathrm{y}$ olivar con formaciones de tomillares y espartales; 3) los páramos formados por calizas lacustres utilizados para cultivos de secano; y 4) los sedimentos fluviales que forman terrazas sobre los cauces fluviales y presentan cultivos de regadío y vegetación de ribera. Además presenta una variabilidad climática ligada al gradiente altitudinal, como pone de manifiesto la variación entre la precipitación media anual que va desde los $1.600 \mathrm{~mm} / \mathrm{año}$ en las cumbres hasta solamente $350 \mathrm{~mm} / \mathrm{año}$ en la depresión.

Debido a la enorme variabilidad ambiental que se recoge en este territorio se ha decidido definir una tipología fluvial específica para esta escala espacial, la Comunidad de Madrid, en lugar de simplemente emplear la existente a nivel estatal, con la que se puede posteriormente contrastar. Para definir esta tipología se ha empleado una metodología semejante a la recomendada por la DMA, (Anexo II sección 1.2 sistema A Directiva 2000/60/CE) en el sentido de considerar las diferentes variables ambientales que afecten a los ríos. Por ello se decidió aprovechar una característica particular del área de estudio, en la Comunidad de Madrid las variables ambientales presentan un patrón de distribución en bandeado de la sierra al llano. La fisiografía refleja nítidamente este patrón de distribución, por ello se puede considerar el resultado de la integración de las variables ambientales. Esta es la razón por la cual se partió del mapa fisiográfico. Al mapa de unidades fisiográficas se le superpone el geológico resumido y se definen las tipologías de río basándose en la litología del lecho fluvial y en la fisiografía.

Para este proceso de definición de tipologías fluviales se ha empleado cartografía digital de la Comunidad de Madrid a una escala de 1:25000. Los programas empleados para la realización del mapa final y la superposición de los ya indicados son el gvGIS y el Arcview.

Una vez definida la tipología de ríos se ubicaron las estaciones consideradas potencialmente de referencia por estar en tramos de ríos naturales o cercanos a la naturalidad. Para decidir la ubicación se cruzaron el mapa de tipologías fluviales con el mapa digital de usos del territorio de la Comunidad de Madrid del año 2000. Las estaciones consideradas potencialmente de referencia se localizaron en zonas de uso "natural" del suelo (si su cuadricula presentaba más de un $70 \%$ de suelo natural (CEDEX, 2004)), sin regulación de caudales aguas arriba y sin recepción de vertidos. Estas condiciones son las que debe cumplir una estación, según la DMA, para poder ser considerada "a priori" como de referencia.

Cada tipología debe contar con varios puntos para recoger la variabilidad de condiciones bióticas y abióticas que se dan de forma natural en cada tipología (Anexo II sección 1.3 Directiva 2000/60/CE; Ortiz Casas, 2002).

\section{Validación de las estaciones preliminares de referencia}

Es imprescindible validar si los puntos considerados como de referencia, en base a la información cartográfica existente a escala regional y de cuenca, pueden formar parte de la red. Lo que obligó a realizar una prospección extensiva de campo para comprobar "in situ" si cumplían las condiciones necesarias, observando si la calidad del agua era buena, si había pocas evidencias de perturbaciones humanas, si el régimen de caudal era natural, si no había cambios significativos sobre el área de drenaje, si el cauce del río tenía condiciones naturales, si había presencia de la vegetación riparia esperada, si no había canales que extrajesen agua del cauce, si el bosque de ribera era autóctono y no presentaba alteraciones importantes y si la calidad del hábitat fluvial era aparentemente buena (Chaves et al., 2006). Se eliminaron las estaciones afectadas por cualquiera de estas alteraciones, cuando afectaban al ecosistema fluvial modificándolo por completo llegando a no ser reconocible como se recoge en la Tabla 3 de resultados. En las restantes estaciones se procedió a realizar una evaluación de su calidad, para lo cual se llevó a cabo una campaña de muestreo realizada en junio-julio de 2008.

Para evaluar las características físicas del hábitat fluvial se aplicó el IHF (Pardo et al., 2002) por considerar este índice un buen evaluador de la heterogeneidad del hábitat fluvial.

La calidad del agua se evaluó analizando distintas variables físico-químicas en campo, $\mathrm{pH}$ 
Tabla 1. Criterios empleados para validar los sitios sin perturbaciones en la Comunidad de Madrid (tomado de Chaves et al., 2006). Criteria used to validate the undisturbed sites in the Community of Madrid (from Chaves et al. 2006).

\begin{tabular}{|c|c|c|c|}
\hline Criterios & Abreviaturas & Descripciones & Bibliografía \\
\hline $\begin{array}{l}\text { Índice de evaluación de la calidad } \\
\text { del agua }\end{array}$ & IBMWP & IBMWP > 100 & $\begin{array}{l}\text { Alba-Tercedor \& Sánchez-Ortega } \\
\text { (1988), Bonada et al. }(2002), \text { Ehler } \\
\text { et al. }(2002)\end{array}$ \\
\hline $\begin{array}{l}\text { Índice de calidad ecológica del } \\
\text { hábitat ripario }\end{array}$ & QBR & $\mathrm{QBR}>66$ & $\begin{array}{l}\text { Munné et al. (2002), Bonada et al. } \\
\text { (2002) }\end{array}$ \\
\hline Índice del hábitat fluvial & IHF & $\mathrm{IHF}>66$ & \\
\hline Parámetros químicos del agua & & $\begin{array}{l}\text { Concentraciones naturales de los } \\
\text { parámetros químicos medidos en el } \\
\text { agua }\end{array}$ & \\
\hline Fósforo total & $\mathrm{TP}$ & $\mathrm{TP}<0.06 \mathrm{mg} / 1$ & \\
\hline Amonio & $\mathrm{NH}_{4}$ & $\mathrm{NH}_{4}<0.13 \mathrm{mg} / 1$ & \\
\hline Nitratos & $\mathrm{NO}_{3}$ & $\mathrm{NO}_{3}<2.96 \mathrm{mg} / \mathrm{l}$ & Adaptado de Bonada et al. (2002) \\
\hline Nitritos & $\mathrm{NO}_{2}$ & $\mathrm{NO}_{2}<0.05 \mathrm{mg} / 1$ & \\
\hline
\end{tabular}

(WTW 320/SET-1), concentración de oxígeno disuelto en $\mathrm{mg} / \mathrm{l}$ y $\%$ y temperatura del agua en ${ }^{\circ} \mathrm{C}$ (WTW 340-B/SET), temperatura del aire en ${ }^{\circ} \mathrm{C}$ (termómetro de mercurio de campo) y conductividad del agua en $\mu \mathrm{s} / \mathrm{cm}$ (WTW LF 340$\mathrm{B} / \mathrm{SET}$ ). Además se tomó una muestra de agua de cada río en botella de plástico que fueron conservadas en frío hasta su procesamiento en el laboratorio el mismo día de su recogida. Este agua, previamente filtrada se analizó mediante distintas técnicas volumétricas y colorimétricas (con ayuda de un espectrofotómetro $\mathrm{HACH}$ DR2010) determinándose la alcalinidad, dureza, cloruros sulfatos, amonio, nitratos, nitritos y fosfatos (APHA-AWWA-WPCF, 1992).

Para evaluar la calidad ecológica de estas estaciones se utilizaron los índices usados y reco- mendados en trabajos como el GUADALMED, realizado para los ríos mediterráneos españoles (Bonada et al., 2002) o en la cuenca del río Mondego en Portugal (Chaves et al., 2006). Para los macroinvertebrados se empleó el índice IBMWP. Las muestras fueron recogidas según la técnica indicada en el PRECE (Jáimez-Cuellar et al., 2002), en el laboratorio se extrajeron manualmente los individuos con ayuda de una lupa binocular, tras lo cual se identificaron hasta nivel de familia empleando claves de determinación (Tachet et al., 2002).

Los índices empleados para evaluar el bosque de ribera fueron el QBR (Munné et al. 1998, 2003) y el RQI (González del Tánago et al., 2006).

En la Tabla 1 se recogen los criterios empleados para realizar la validación de los sitios con-

Tabla 2. Estaciones preliminares de referencia en relación de las distintas tipologías fluviales de la Comunidad de Madrid. Preliminary reference stations in relation to the different river typologies of the Community of Madrid.

\begin{tabular}{|c|c|c|}
\hline Tipologías de ríos & $\begin{array}{c}\text { Superficie de territorio } \\
\text { ocupado }(\%)\end{array}$ & $\begin{array}{l}\text { Número de estaciones } \\
\text { preseleccionadas }\end{array}$ \\
\hline Ríos calcáreo-arcillosos en interfluvios y vertientes & 23.86 & 3 \\
\hline Ríos silíceos en pie de monte & 23.19 & 5 \\
\hline Ríos montañoso silíceos & 16.58 & 3 \\
\hline Ríos calcáreo-arcillosos en páramos y alcarrias & 11.72 & \\
\hline Ríos calcáreo-arcillosos en campiña & 7.22 & \\
\hline Ríos en terrazas y llanuras aluviales & 5.28 & 4 \\
\hline Ríos calcáreo arcillosos en lomas y campiñas & 3.17 & \\
\hline Ríos silíceos en interfluvios y vertientes & 2.72 & \\
\hline Ríos calcáreo-arcillosos en pie de monte & 1.76 & 1 \\
\hline Ríos en relieves de transición de la cuenca & 1.51 & \\
\hline Ríos calcáreo-arcillosos en montaña & 0.07 & \\
\hline
\end{tabular}


siderados preliminarmente de referencia por no presentar impactos. Estos criterios han sido utilizados por otros autores (Chaves et al., 2006) además recogen parámetros relativos a la concentración de nutrientes y se adaptan mejor a las condiciones de nuestro área de estudio que los establecidos por el Ministerio de Medio Ambiente.

También se ha decidido emplear el RQI para validar los sitios considerados potencialmente de referencia. Con un RQI superior a 79 se considerará que el bosque de ribera no está alterado (González del Tánago et al., 2006).

Para el fósforo se va a utilizar el ión ortofosfato o fósforo reactivo soluble (PRS) por considerarse más adecuado. Un punto de referencia sería aquél que presentase un nivel de fosfato menor a $0.05 \mathrm{mg} / \mathrm{l}\left(\mathrm{P}-\mathrm{PO}_{4}^{3-}\right)$. (Bonada et al., 2002).

Los estadillos empleados en la campaña de muestreo y caracterización de los puntos de referencia establecidos preliminarmente sobre el mapa de la Comunidad de Madrid son iguales a los empleados en el proyecto GUADALMED (Jáimez-Cuéllar et al., 2002).

\section{RESULTADOS}

En la Comunidad de Madrid se definieron 11 tipos fluviales que se exponen en la Tabla 2 y cuya localización junto con la de las estaciones de re-
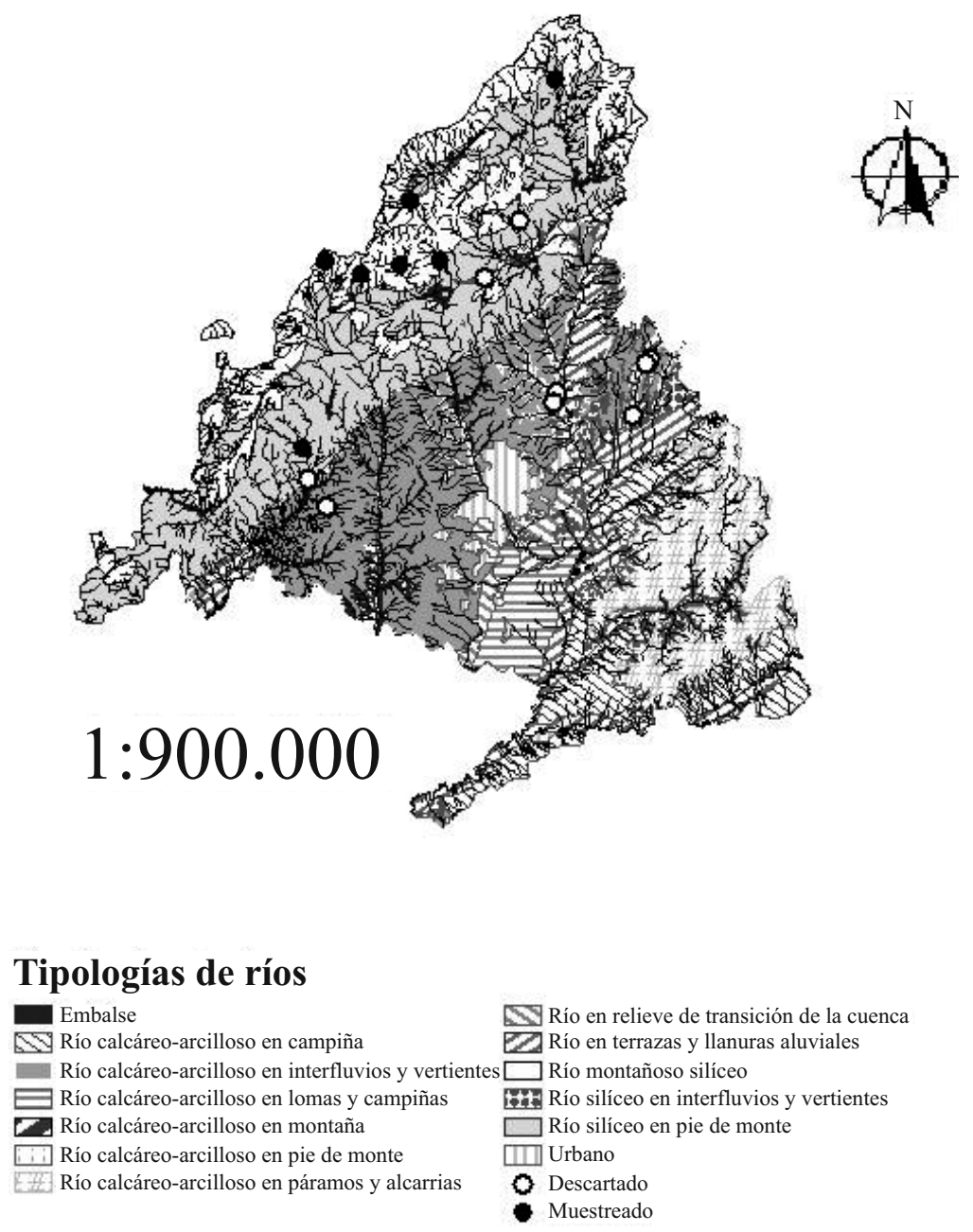

Figura 1. Mapa de tipologías de los ríos de la Comunidad de Madrid. Map of the river typologies in the Community of Madrid. 
Tabla 3. Caracterización básica de las estaciones de referencia preliminares. Basic characterization of the preliminary reference stations.

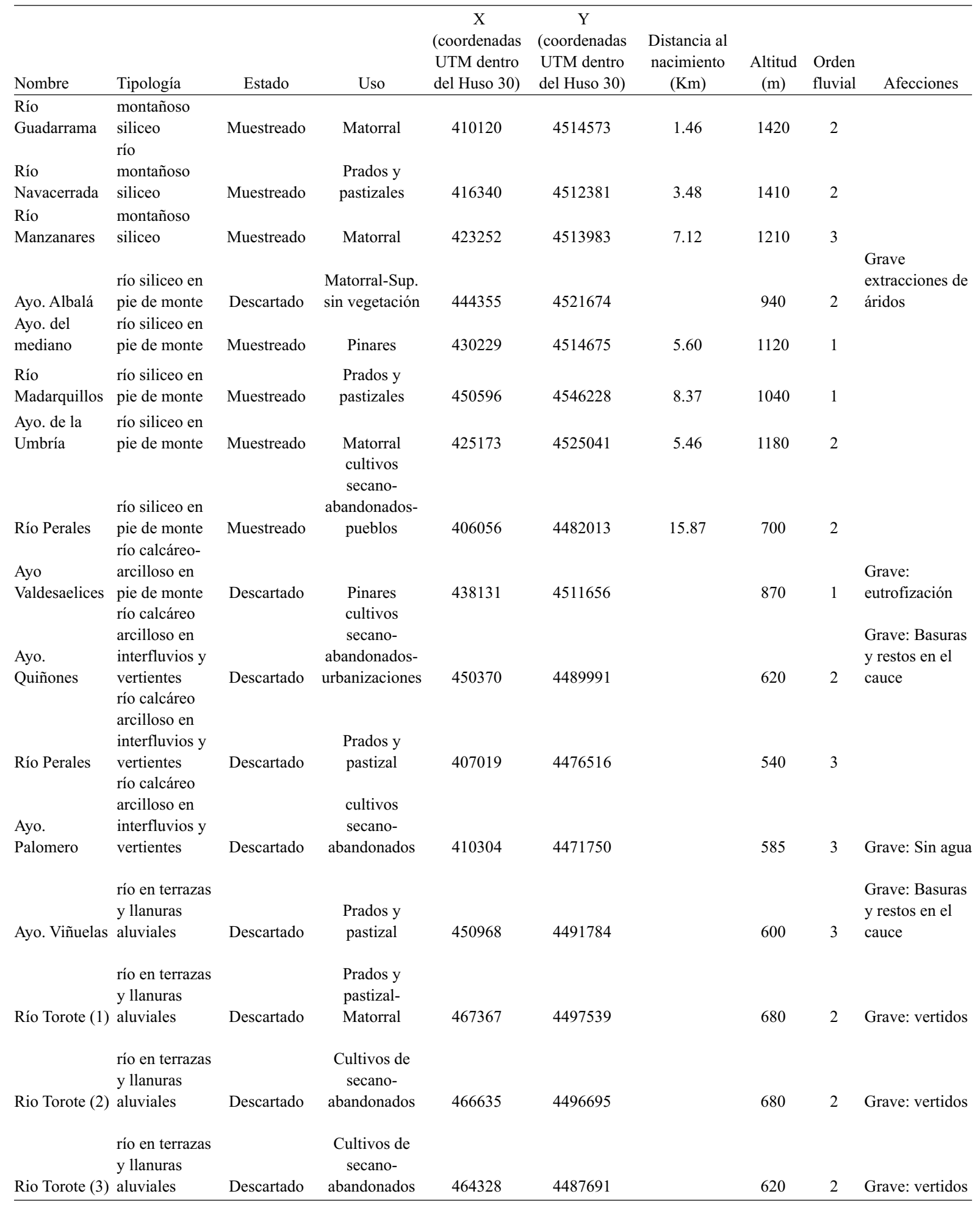


ferencia se puede ver en la figura 1. La tipología de un río va a condicionar la fauna de macroinvertebrados que presente, las características hidroquímicas, comunidades y funcionalidad del río.

A partir de este mapa se han calculado los porcentajes de territorio que ocupan cada una de las tipologías (Tabla 2). Para tener representadas todas las tipologías y con el gradiente de variación que exige la DMA, la red de referencia debería contar aproximadamente con 27 estaciones. Si todos los ríos presentasen condiciones naturales deberían disponerse 3 puntos en las tipologías con mayor representatividad y 2 en el resto. Tras incorporar la información del mapa de usos del suelo de la Comunidad de Madrid (2000) y descartar los tramos de río situados por debajo de embalses y los que discurrían por terreno urbanizado o de cultivo, únicamente fue posible seleccionar y disponer sobre el mapa 16 estaciones. Su localización y características se detallan en la Tabla 3. Sólo en 5 de las 11 tipologías pudimos situar estaciones que cumplieran los requerimientos necesarios. Esto da idea del fuerte estado de deterioro de una gran parte del territorio de la Comunidad de Madrid. Los ríos en los que ha sido imposible si- tuar estaciones de referencia suponen 6 tipologías y más del $26 \%$ de la superficie del territorio.

Tras la campaña de prospección extensiva y muestreo se observó que sólo 7 puntos cumplían los requisitos de la DMA, ya que 9 de los 16 presentaban afecciones locales que los invalidaban (Tabla 3). Únicamente se dispuso de estaciones en 2 de las 11 tipologías de río que existen en el área de estudio, ríos silíceos en pie de monte y ríos montañosos silíceos. En los ríos montañosos silíceos se han muestreado 3 estaciones y en los silíceos de pie de monte 4 , dado que el porcentaje de territorio que ocupan es mayor.

Los valores de los parámetros físico-químicos de calidad del agua se presentan en la Tabla 4. Las aguas de los ríos estudiados son frías. Su mineralización es débil lo que se observa por los bajos niveles de conductividad. El Perales y el Umbría presentan los valores más elevados de $\mathrm{pH}$ y alcalinidad. Para el resto de los ríos se observa que los valores de $\mathrm{pH}$ están próximos a la neutralidad. El porcentaje de oxígeno disuelto es elevado, próximo o ligeramente superior a la saturación, excepto en el Perales, río del pie de monte silíceo con mayor temperatura del agua y corriente menos turbulenta.

Tabla 4. Parámetros físico-químicos medidos en las estaciones seleccionadas como potencialmente de referencia. Physicalchemical variables measured in the stations selected as potentially reference stations.

\begin{tabular}{|c|c|c|c|c|c|c|c|}
\hline Variables medidas & $\begin{array}{c}\text { Río } \\
\text { Guadarrama }\end{array}$ & $\begin{array}{c}\text { Río } \\
\text { Navacerrada }\end{array}$ & $\begin{array}{c}\text { Río } \\
\text { Manzanares }\end{array}$ & $\begin{array}{c}\text { Ayo. de la } \\
\text { Umbría }\end{array}$ & $\begin{array}{l}\text { Ayo. del } \\
\text { Mediano }\end{array}$ & $\begin{array}{c}\text { Río } \\
\text { Madarquillos }\end{array}$ & $\begin{array}{c}\text { Río } \\
\text { Perales }\end{array}$ \\
\hline Tipología & \multicolumn{3}{|c|}{ ríos montañosos siliceos } & \multicolumn{4}{|c|}{ ríos siliceos en pie de monte } \\
\hline Fecha de muestreo & 03/07/08 & 03/07/08 & 04/07/08 & $30 / 06 / 08$ & 04/07/08 & $30 / 06 / 08$ & $26 / 06 / 08$ \\
\hline $\mathrm{T}^{\circ}$ aire $\left({ }^{\circ} \mathrm{C}\right)$ & & & 20.4 & 24.5 & 24 & 30 & 32 \\
\hline $\mathrm{T}^{\circ}$ agua $\left({ }^{\circ} \mathrm{C}\right)$ & 11 & 9.5 & 11.8 & 16.7 & 15 & 17.7 & 22.2 \\
\hline Conductividad $(\mu \mathrm{S} / \mathrm{cm})$ & 25.8 & 19.9 & 16.8 & 149 & 23.5 & 49 & 303.4 \\
\hline $\mathrm{pH}$ & 7.1 & 6.6 & 6.6 & 7.8 & 6.6 & 7.1 & 8.3 \\
\hline Oxígeno disuelto (mg/l) & 9.26 & 10.24 & 9.36 & 9.2 & 8.74 & 8.78 & 5.2 \\
\hline Saturación de oxígeno (\%) & 99 & 99.9 & 99.6 & 106.4 & 94.5 & 103.2 & 64 \\
\hline Alcalinidad $\left(\mathrm{mg} / 1 \mathrm{CO}_{3} \mathrm{Ca}\right)$ & 15.2 & 7.1 & 5.1 & 73.8 & 9.1 & 6.6 & 100.9 \\
\hline Dureza $\left(\mathrm{mg} / 1 \mathrm{CO}_{3} \mathrm{Ca}\right)$ & 11.5 & 6.2 & 5 & 79.4 & 8.6 & 21.2 & 79.5 \\
\hline Cloruros (mg/l) & 5.1 & 1.8 & 1.1 & 4.1 & 5.3 & 10.9 & 33.1 \\
\hline Sulfatos (mg/l) & 0 & 1 & 1 & 8 & 3 & 1 & 16 \\
\hline Nitratos (mg/l) & 0.1 & 0.2 & 0.3 & 0.18 & 0.2 & 0.23 & 0.1 \\
\hline Nitritos (mg/l) & 0.002 & 0.004 & 0.004 & 0.01 & 0.002 & 0.012 & 0.01 \\
\hline Amonio (mg/l) & 0.01 & 0.01 & 0.01 & 0.01 & 0.01 & 0.03 & 0.19 \\
\hline Fosfatos (mg/l) & 0.17 & 0.13 & 0.17 & 0.05 & 0.12 & 0.2 & 1.86 \\
\hline
\end{tabular}


Tabla 5. Valores del índice de hábitat fluvial (IHF), de los índices y número de familias de macroinvertebrados (IBMWP, IASPT) y de los índices de calidad de ribera (QBR, RQI). Values of the fluvial habitat index (IHF), the indexes, and number of macroinvertebrate families (IBMWP, IASPT), and the riverbank quality indexes $(Q B R, R Q I)$.

\begin{tabular}{|c|c|c|c|c|c|c|c|}
\hline & Río Guadarrama & Río Navacerrada & Río Manzanares & Ayo. de la Umbría & Ayo. del mediano & Río Madarquillos & Río Perales \\
\hline IHF & 68 & 70 & 74 & 78 & 77 & 78 & 47 \\
\hline IBMWP & 158 & 176 & 145 & 213 & 158 & 154 & 120 \\
\hline EQR & 0.88 & 0.98 & 0.81 & 1.18 & 0.88 & 0.86 & 0.70 \\
\hline IASPT & 6.08 & 6.28 & 6.04 & 6.08 & 6.58 & 5.70 & 4.80 \\
\hline N. ${ }^{\circ}$ FAMILIAS & 26 & 28 & 24 & 35 & 24 & 27 & 25 \\
\hline QBR & 60 & 60 & 85 & 75 & 70 & 80 & 45 \\
\hline RQI & 112 & 86 & 100 & 98 & 98 & 111 & 68 \\
\hline
\end{tabular}

Presentan una carga de nutrientes baja, los nitratos y nitritos no sobrepasan los valores máximos establecidos para que una estación pueda ser considerada de referencia. Para el amonio sólo la estación ubicada en el Perales sobrepasa el valor límite para ser considerada de referencia. En el caso del fosfato, aunque los valores son en general bajos excepto en el Perales, sólo el arroyo Umbría presenta una concentración menor de $0.05 \mathrm{mg} / \mathrm{l}$, condición establecida para considerar que una estación es de referencia.

En la Tabla 5 aparecen los valores de los índices de calidad del hábitat, vegetación de ribera y de macroinvertebrados. La calidad del ambiente físico del cauce es buena en las estaciones de los ríos Guadarrama, Navacerrada, Manzanares, Madarquillos y del arroyo Umbría y Mediano, por presentar valores del índice IHF superiores a 66. En relación a este índice 6 de las 7 estaciones pueden considerarse de referencia.

A partir de los valores de IHF es posible realizar una predicción de los valores de IBMWP y la riqueza de familias. El Manzanares, el Navacerrada, el Madarquillos, el arroyo Umbría y el Mediano se corresponderían con una calidad biológica mayor de 120 y de hasta 250 y una riqueza de familias entre 25 y 45 (Pardo $e t$ al., 2002). El Madarquillos, el Navacerrada y el Umbría, cumplen la predicción, presentando una riqueza de familias de 27, 28 y 35 y un IBMWP superior a 120. El Manzanares y el Mediano cumplen la predicción para el IBMWP pero no para la riqueza de familias porque es inferior a 25. Para el Guadarrama según el valor de IHF se predice un valor de IBMWP de entre 80 y 220 y una riqueza de familias entre 19 y 37 , y de forma coherente presenta 26 familias y el valor del índice es
158. El Perales presenta un valor IHF que le permite sostener no más de 26 familias y una calidad biológica inferior a 120 (Pardo et al., 2002) y presenta 25 familias y un valor de IBMWP de 120.

Las 7 estaciones muestreadas presentan un amplio rango de valores de IBMWP. Únicamente el Arroyo Umbria supera el valor de 180 considerado oficialmente como condición de referencia para ríos tipo 11 , todos los demás son ligeramente inferiores pero superan el límite muy bueno/bueno. Según el índice IASPT los ríos se corresponden con una buena o muy buena calidad (Confederación Hidrográfica del Ebro, 1999).

Las clases de calidad del bosque de ribera, en algunos casos, dependen del índice utilizado. Las estaciones de Navacerrada, Guadarrama y Mediano presentan respectivamente un valor de QBR de 60, 60 y 70 (calidad regular), sin embargo el valor obtenido con el índice RQI fue de 86,112 y 98 , lo que se corresponde con un bosque de galería de buena calidad. Mientras que el río Manzanares, Arroyo Umbría, río Madarquillos y río Perales, las clases de calidad son las mismas con ambos índices, siendo buena excepto en el caso del Perales.

\section{DISCUSIÓN}

Aplicando la tipología oficial (Directiva 2000/ $60 / \mathrm{CE}$ ), en la comunidad de Madrid solamente aparecen 6 tipos, de los cuales 5 (ríos manchegos, ríos de baja montaña mediterránea silícea, ríos de montaña mediterránea calcárea, ejes mediterráneo-continentales poco mineralizados y grandes ejes en ambiente mediterráneo) o solo recogen un único pequeño arroyo sin importancia o 
se trata de los tramos finales altamente contaminados de los ejes principales. Por ello, solamente podríamos considerar 1 única tipología mayoritaria: tipo 11 ríos de montaña mediterránea silícea, que evidentemente no contempla la variabilidad ambiental existente a esta escala espacial.

Hay distintos métodos de diferenciación de tipologías fluviales, en este estudio para definir la tipología de ríos de la Comunidad Autónoma de Madrid se ha empleado la cartografía digital existente. En la mayoría de los estudios del mismo tipo se realizan análisis de componentes principales (ACP) empleando las variables clima, litología, geomorfología e hidrografía expresadas en forma de variables cuantitativas (Roblas et al. 2005). Sin embargo, comparando los resultados de este estudio con los del estudio de definición de tipologías realizado para la cuenca del Guadarrama mediante ACP (Roblas et al. 2005), se obtienen básicamente las mismas tipologías. Esto parece indicar que la metodología empleada en este estudio para definir las tipologías fluviales existentes en la comunidad de Madrid es también correcta.

Es útil realizar una selección previa de estaciones de referencia sobre cartografía porque permite integrar toda la información subyacente a la heterogeneidad del territorio que de otra manera sería imposible o muy costoso tener en cuenta. Además se evita recorrer el área de estudio en su totalidad, si bien es cierto que debe complementarse con visitas de campo. En otros proyectos similares también se realiza esta fase de selección previa de estaciones sobre cartografía (Chaves et al. 2006) utilizando el mapa de usos del suelo y distinguiendo uso natural y modificado (CEDEX, 2004; Chaves et al. 2006; Artemiadou et al. 2008).

En la Comunidad de Madrid existen problemas para establecer una red de referencia debido a la alta densidad y concentración poblacional (6.386.932 habitantes y 798 habitantes/ $\mathrm{Km}^{2}$ ) y a la extensión de terreno urbanizado (alrededor del $10 \%$ del territorio) lo que da lugar a una grave alteración y modificación de las redes fluviales. Por lo que sólo se han podido disponer 16 estaciones preliminares de referencia sobre la cartografía digital de la Comunidad. En torno a un $60 \%$ de los ríos de la comunidad no pueden con- tar con estaciones de referencia, y sólo 2 de las 11 tipologías definidas en este estudio han podido ser recogidas en la red preliminar, con 7 estaciones potenciales. Para los tramos fluviales que presentan grandes obras hidráulicas sería adecuado definirlos como masas de agua muy modificadas y definir máximos potenciales ecológicos según la orden ARM/2656/2008 de 10 de septiembre por la que se aprueba la instrucción de planificación hidrológica.

Utilizando los criterios de validación de estaciones de referencia (Tabla 1) en las 7 estaciones que cumplen los requisitos de selección, observamos que se obtienen distintos resultados según los diferentes parámetros e índices considerados. En cuanto a las características físico-químicas del agua, el río Perales presenta una elevada concentración de amonio y fosfatos, por lo que ésta es la única estación que no podría ser considerada. Para la concentración de fosfato en el agua sólo la estación ubicada en el Ayo. Umbría presenta menos de $0.05 \mathrm{mg} / \mathrm{l}$ lo que permite considerarla de referencia (Bonada et al, 2002), aunque este valor se supera en todas las demás estaciones sus concentraciones siempre se mantienen en un rango muy bajo que no supone un deterioro de la calidad ecológica del río. Aún así habría que comprobar si este patrón es extensible a todos los ríos del área de estudio de la tipología montañoso silícea y en caso de ser así, establecer un nuevo límite para la concentración de fosfato que de forma natural se registra en estos ríos de la Sierra de Guadarrama.

Los criterios oficiales para los parámetros físico-químicos sólo consideran el oxígeno disuelto, la conductividad y el $\mathrm{pH}$, y no tienen en cuenta los nutrientes, parámetros que consideramos mucho más importantes. Todos los ríos estudiados cumplen los valores establecidos para los dos primeros parámetros pero respecto al $\mathrm{pH}$ ninguno de los 6 ríos de tipo 11 (valor de referencia 8.1) llega a alcanzar este límite, por lo que oficialmente ninguna de estas estaciones se podría validar como de referencia. No obstante este valor de referencia nos parece extremadamente alto para ríos que discurren sobre sustrato silíceo y desde nuestro punto de vista no debería ser considerado para descartar estas estaciones. 
La calidad del ambiente físico del cauce (IHF) es buena en todas las estaciones excepto en el Perales si utilizamos el valor de corte de 66 recomendado por Pardo et al. (2002), sin embargo si utilizamos el valor oficial (72) Manzanares, Umbría, Mediano y Madarquillos serían de referencia, Guadarrama y Navacerrada estarían en el percentil 0.92 y únicamente Perales sería descartada. El IHF es un índice bastante valido en nuestros ríos para predecir los valores esperables de IBMWP y número de familias, ya que sólo en dos estaciones (Manzanares y Ayo. Mediano) no se cumple la predicción para el número de familias, aunque sí para el valor de IBMWP.

Según el índice IBMWP y utilizando el valor de corte de 100 (Alba-Tercedor \& SanchezOrtega, 19988; Bonada et al., 2002; Ehler et al., 2002; Jaimez-Cuéllar et al., 2002) las 7 estaciones muestreadas se pueden considerar de referencia por presentar un valor muy superior. Oficialmente el valor considerado como de referencia para ríos tipo 11 es de 180. Únicamente el Arroyo Umbría lo supera, todas las demás estaciones excepto el Perales presentan un EQR muy superior a 0.78 que es el primer percentil y permite caracterizar a las estaciones por encima del límite muy bueno/bueno, por lo que pensamos que podrían ser consideradas todas ellas como de referencia.

El IASPT es un índice poco empleado para valorar la calidad del agua de los ríos pese a ello, hemos decidido utilizarlo ya que este índice detecta cambios de calidad que con IBMWP quedan ocultos (Oscoz et al., 2005). El IASPT se correlaciona negativamente con la concentración de amonio y fosfatos de manera más significativa que el IBMWP (Bordallo, 2009). Todas las estaciones muestreadas presentan valores elevados de este índice, por lo que se trata de estaciones con muy buena calidad (Confederación Hidrográfica del Ebro).

En cuanto a los índices de calidad de ribera el cálculo del QBR en las estaciones de Navacerrada y Manzanares no está recomendado por los autores del mismo (Munné et. al, 1998), ya que ambas estaciones se encuentran en una zona con un porcentaje de sustrato duro mayor al $80 \%$. Utilizando este índice las estaciones ubicadas en el Manzanares, en el Umbría y en el Madarqui- llos, pueden ser consideradas de referencia con valores de corte >75 (Munné et al, 1998, 2003; Bonada et al., 2002). El valor de corte oficial es de 87.5 por lo que según este criterio se deberían descartar todas las estaciones.

Con el índice RQI la única estación descartada sería la ubicada en el Perales por presentar un valor inferior a 79 (Gónzalez del Tánago et $a l ., 2006)$. En este caso parece ser más correcto emplear el RQI para evaluar la calidad del bosque de galería ya que se puede aplicar en todas las estaciones, incluidos los tramos abruptos de cabecera y, los resultados obtenidos con él están más acordes con las características concretas de los bosques de galería observados en campo en cada uno de los ríos estudiados.

Estos resultados muestran que 6 de los 7 puntos estudiados podrían formar parte de la red de referencia si empleamos nuestros criterios de validación recogidos en la Tabla 1 . Se tendrían representadas dos tipologías, los ríos silíceos en pie de monte y los montañosos silíceos del Sistema Central, para las cuales se podrían localizar respectivamente 4 y 3 estaciones de referencia. Los ríos montañoso silíceos del Sistema central son pequeños arroyos de montaña situados a elevadas altitudes, muy encajados, estrechos, poco profundos, con grandes afloramientos de roca madre, poco desarrollo del medio hiporreico, escaso caudal y fuerte torrencialidad, junto con una marcada fluctuación estacional del caudal, incluso estacionalidad. Además presentan un escaso desarrollo de la vegetación de ribera propiamente dicha por la inexistencia de orillas aluviales. Por el contrario los ríos silíceos en pie de monte tienen pendientes inferiores, valles más amplios y mayor desarrollo aluvial, por lo que presentan una zona adecuada para que se pueda desarrollar una vegetación riparia y un medio hiporreico, de mayor entidad que lleva a que los caudales de estos segundos sean permanentes durante todo el año y más elevados. Por ello se ha decidido definir éstas dos tipologías en lugar de emplear la oficial en la cual todos los ríos estudiados se corresponderían con el tipo 11 .

La única estación descartada por incumplir la mayor parte de los parámetros físico-químicos e índices considerados sería la del río Perales. $\mathrm{Cu}$ - 
riosamente esta estación es considerada de referencia para los ríos de baja montaña mediterránea silícea según los datos de la Confederación Hidrográfica del Tajo para el índice biótico de diatomeas IPS, empleando los valores establecidos para tal fin en la ARM/ 2656/ 2008.

A pesar del objetivo del trabajo de encontrar diferentes tipologías para la zona estudiada, no contamos con suficientes estaciones de referencia para la mayoría de las tipologías fluviales predeterminadas dentro de la Comunidad de Madrid. Estos resultados muestran la limitación del espacio territorial escogido y que sería conveniente ampliar el área de estudio a la cuenca del Tajo, esto nos permitiría ajustar los valores de corte de ríos inalterados a las condiciones de estos ríos y dar rangos de calidad.

\section{BIBLIOGRAFÍA}

\section{ALBA-TERCEDOR, J. \& A. SÁNCHEZ-ORTEGA.} 1988. Un método rápido y simple para evaluar la calidad biológica de las aguas corrientes basado en el de Hellawell (1978). Limnetica, 4: 51-56.

APHA-AWWA-WPCF. 1992. Standard Methods for the Examination of Water and Wastewater. 18th. Edition. U.S.A. 1325 pp.

ARTEMIADOU, V., X. STATIRI, TH. BROUZIOTIS \& M. LAZARIDOU. 2008. Ecological quality of small mountainous Mediterranean streams (river type R-M4) and performance of the European intercalibration metric. Hydrobiologia, 605(1): 7588.

BONADA, N., N. PRAT, A. MUNNÉ, M. RIERADEVALL, J. ALBA-TERCEDOR, M.ÁLVAREZ, J. AVILÉS, J. CASAS, P. JÁIMEZ-CUÉLLAR, ANDRÉS MELLADO, G. MOYÁ, I. PARDO, S. ROBLES, G. RAMÓN, M. L. SUÁREZ, M. TORO, M. R. VIDAL-ABARCA, S. VIVAS \& C. ZAMORA-MUÑOZ. 2002a. Ensayo de una tipología de las cuencas mediterráneas del proyecto GUADALMED siguiendo las directrices de la directiva marco del agua. Limnetica, 21 (3-4): 77-98.

BONADA, N., N. PRAT, A. MUNNÉ, M. RIERADEVALL, J. ALBA-TERCEDOR, M.ÁLVAREZ, J. AVILÉS, J. CASAS, P. JÁIMEZ-CUÉLLAR, ANDRÉS MELLADO, G. MOYÁ, I. PARDO, S. ROBLES, G. RAMÓN, M. L. SUÁREZ, M. TORO,
M. R. VIDAL-ABARCA, S. VIVAS \& C. ZAMORA-MUÑOZ. 2002b. Criterios para la selección de condiciones de referencia en los ríos mediterráneos. Resultados del proyecto GUADALMED. Limnetica, 21(3-4): 99-114.

BORDALLO, A. 2009. Diseño de una red de referencia para la implantación de la Directiva Marco del Agua en la Comunidad de Madrid. Trabajo fin de Máster. Máster Oficial en Ecología. Dept. de Ecología. Universidad Autónoma de Madrid. 42 pp.

CARRANZA, M. L. \& J. ARÍSTEGUI, 2002. Ríos de Madrid Naturaleza e Historia. Ed. Consejería de Medio ambiente (Comunidad de Madrid) y Ediciones Mundi-Prensa. $167 \mathrm{pp}$.

CEDEX. 2004. Selección preliminar de posibles tramos fluviales de referencia. Versión 1.0. Centro de estudios y experimentación de obras públicas, Ministerio de Fomento, Madrid. 20 pp.

CEDEX. 2005. Caracterización de los tipos de ríos y lagos. Versión 4.0. Centro de Estudios Hidrográficos, Ministerio de Fomento, Madrid. 219 pp.

CHAVES, M. L., J. L. COSTA, P. CHAINHO, M. J. COSTA, \& N. PRAT, 2006. Selection and validation of reference sites in small river basins. Hydrobiologia, 573: 133-154.

CONFEDERACIÓN HIDROGRÁFICA DEL EBRO. 1999. Delimitación de regiones ecológicas en la cuenca del Ebro. Departamento de Ecología, Universidad de Barcelona. $152 \mathrm{pp}$.

EHLERT, T., D. HERING, U. KOENZEN, T. POTTGIESSER, H. SCHUHMACHER \& G. FRIEDRICH, 2002. Typology and type specific reference conditions for medium sized and large rivers in Northrhine-Westphalia: Methodological and biological aspects. International Review of Hydrobiology, 87: 151-163.

GONZÁLEZ DEL TÁNAGO, M., D. GARCÍA DE JALÓN, F. LARA \& R. GARILLETI. 2006. Índice RQI para la valoración de las riberas fluviales en el contexto de la directiva marco del agua. Ingeniería Civil, 143: 97-108.

GONZÁLEZ DEL TÁNAGO, M. \& D. GARCÍA DE JALÓN. 2006. Propuesta de caracterización jerárquica de los ríos españoles para su clasificación según la Directiva Marco del Agua. Limneti$c a, 25(3)$ : 693-712.

JÁIMEZ-CUÉLLAR, P., S. VIVAS, N. BONADA, S. ROBLES, A. MELLADO, M. ÁLVAREZ, J. AVILÉS, J. CASADO, M. ORTEGA, I. PARDO, N. PRAT, M. RIERADEVALL, C. M. SÁINZ-CANTERO, A. SÁNCHEZ-ORTEGA, M. L SUÁ- 
REZ, M. TORO, M. R. VIDAL-ABARCA, C. ZAMORA-MUÑOZ \& J. ALBA-TERCEDOR. 2002. Protocolo Guadalmed (PRECE). Limnetica, 21(34): 187-204.

LARA, F. 2005. Formaciones vegetales de ribera en España. Implicaciones para la restauración. En: II Curso sobre Ecología fluvial y restauración de ríos $y$ riberas. CEDEX (Ed.). 18-33. Ministerio de Fomento, Madrid.

MUNNÉ, A., C. SOLÀ \& N. PRAT, 1998. QBR: Un índice rápido para la evaluación de la calidad de los ecosistemas de ribera. Tecnología del Agua, 175: 20-37.

MUNNÉ, A., N. PRAT, C. SOLÀ, N. BONADA \& M. RIERADEVALL, 2003. A simple field method for assessing the ecological quality of riparian habitat in rivers and streams. QBR index. Aquatic Conservation: Marine and Freshwater Ecosystems, 13: 147-164.

ORTIZ CASAS, J. L. 2002. La directiva marco del agua (2000/60/CE): aspectos relevantes para el proyecto GUADALMED. Limnetica, 21 (3-4): 5-12.

OSCOZ, J., P. M. LEUNDA., R. MIRANDA \& M. C. ESCALA., 2005. Calidad biológica de las aguas en el río Erro (Navarra, N España) (2001-2002). Ecología, 19: 59-74.

PARDO, I., ÁlVAREZ, M., CASAS, J., MORENO,
J. L., VIVAS, S., BONADA, N., ALBA-TERCEDOR, J., JÁIMEZ-CUÉLLAR, P., MOYÁ, G., PRAT, N., ROBLES, S., SUÁREZ, M. L., TORO, M. \& VIDAL-ABARCA, M. R. 2002. El hábitat de los ríos mediterráneos. Diseño de un índice de diversidad de hábitat. Limnetica, 21(3-4): 115-133. RIVAS-MARTÍNEZ, S., T. E. DÍAZ, F. FERNÁNDEZ-GONZÁLEZ, J. IZCO, J. LOIDI, M. LOUSA \& A. PENAS, 2002. Vascular plant communities of Spain and Portugal. Addenda to the syntaxonomical checklist of 2001. Part I. Itinera Geobotanica, 15 (1): 5-432.

ROBLAS, N., C. ROZAS, J. GARCÍA, 2005. Ecosistemas fluviales secundarios de la cuenca del río Guadarrama en la Comunidad de Madrid. Tipología, calidad biológica e incidencia de la actividad humana en su conservación. Centro de Investigaciones Ambientales de la Comunidad de Madrid "Fernando González Bernáldez". Informe CIAM/1818. $92 \mathrm{pp}$.

ROSGEN, D. 1996. Applied River Morphology. Wildland Hydrology, Pagosa Springs, Colorado, USA. 385 pp.

TACHET, H., P. RICHOUX, M. BOURNAUD \& P. USSEGLIO-POLATERA, 2002. Invertebres d'eau douce systematique, biologie et écologie. CNRS Editions, Paris. 587 pp. 